\title{
Antiplatelet Therapy Use and the Risk of Venous Thromboembolic Events in the Raloxifene Use for the Heart (RUTH) Trial
}

\author{
Claire S. Duvernoy, M.D., Adeline A. Yeo, M.S., ${ }^{2}$ Mayme Wong, Ph.D., \\ David A. Cox, Ph.D., ${ }^{2}$ and Hyungjin M. Kim, Sc.D. ${ }^{3}$
}

\begin{abstract}
Background: Raloxifene use in postmenopausal women with osteoporosis increases the risk of venous thromboembolic events (VTE) 2-fold compared with placebo. Platelet activation is involved in the pathophysiology of arterial thromboses more than venous thromboses, but aspirin may reduce VTE risk associated with estrogen use. This analysis examines the effects of concomitant antiplatelet therapy on VTE risk in raloxifene-treated women.

Methods: In the Raloxifene Use for the Heart (RUTH) trial, 10,101 postmenopausal women from 177 sites in 26 countries at increased risk of coronary heart disease (CHD) (primary prevention cohort) or with CHD (secondary prevention cohort) were randomized to placebo or raloxifene $60 \mathrm{mg}$ /day and followed for a median 5.6 years. Reports of clinical symptoms of VTE were assessed. Concomitant use of antiplatelet agents (aspirin, clopidogrel, ticlopidine, dipyridamole) was allowed. Cox proportional hazard models, with use of warfarin, presence of fracture, and hospitalization as covariates, were used to estimate hazard ratios (HR) with $95 \%$ confidence intervals (CI).

Results: Overall, raloxifene use was associated with an increased VTE risk (HR 1.44, 95\% CI 1.06-1.95) vs. placebo. Most women (72\%) reported using aspirin, and $14.2 \%$ reported using nonaspirin antiplatelet agents during the study period. Users of antiplatelet agents were older, more likely to have CHD, and more likely to be hyperlipidemic. They had a higher VTE risk than nonusers. No difference in VTE risk was observed in women who used raloxifene alone vs. those who used raloxifene with antiplatelet agents during the study. The increase in VTE risk with raloxifene compared with placebo was not different between women who used antiplatelet agents at baseline (HR 1.44, 95\% CI 0.98, 2.10) and those who did not use antiplatelet agents (HR 1.37, 95\% CI $0.83,2.27$ ) (interaction $p=0.88$ ). Similar conclusions were noted for aspirin and nonaspirin antiplatelet use.

Conclusions: In RUTH, postmenopausal women treated with raloxifene had an increased risk of VTE compared with placebo. Concomitant use of aspirin or nonaspirin antiplatelet agents along with raloxifene did not change VTE risk.
\end{abstract}

\section{Introduction}

$\mathbf{R}$ ALOXIFENE IS A SELECTIVE estrogen receptor modulator (SERM) that is indicated for osteoporosis prevention and treatment in postmenopausal women and for the reduction in risk of invasive breast cancer in postmenopausal women with osteoporosis or at high risk for invasive breast cancer. ${ }^{1}$ As with other SERMs and estrogen itself, ${ }^{2}$ the use of raloxifene is associated with a significantly increased risk of venous thromboembolism (VTE) compared with placebo. ${ }^{3-5}$ The mechanism of action for this increased risk is not fully understood but likely is multifactorial and includes acquired resistance to activated protein $C$, such prothrombotic effects as decreased protein $\mathrm{S}$ and antithrombin III levels, and increased prothrombin fragments $1+2$, fibrinopeptide A, and factor VII. ${ }^{6}$ The increased hepatic synthesis of inflammatory markers may also be a VTE risk factor for oral estrogens. $^{7}$

\footnotetext{
${ }^{1}$ VA Ann Arbor Healthcare System/University of Michigan, Ann Arbor, Michigan.

${ }^{2}$ Eli Lilly and Company, Indianapolis, Indiana.

${ }^{3}$ University of Michigan, Ann Arbor, Michigan.
} 
In the Heart and Estrogen/progestin Replacement Study (HERS), a randomized trial comparing conjugated equine estrogen (CEE) plus medroxyprogesterone acetate (MPA) vs. placebo for the prevention of coronary heart disease (CHD) events in women with prior CHD, concomitant use of aspirin with the study drug caused a 50\% risk reduction in VTEs compared with women taking the study drug who were not using aspirin. 3,8 Alternatively, other analyses from the Women's Health Initiative (WHI) trial did not show any significant effect of aspirin use on the increased risk of VTE with CEE alone or when combined with $\mathrm{MPA}^{9}$ compared with placebo. These observations led us to investigate whether the increased risk of VTEs with raloxifene use over placebo differed between antiplatelet agent users and nonusers; that is, did antiplatelet use lower the risk of VTE with raloxifene? The Raloxifene Use for the Heart (RUTH) trial was designed to determine if the use of raloxifene lowered cardiovascular event rates or breast cancer occurrence in postmenopausal women at increased risk for cardiovascular events. This analysis sought to determine whether antiplatelet use by women enrolled in RUTH lowered the risk of VTE associated with raloxifene use in the trial.

\section{Materials and Methods}

RUTH was a prospective, randomized, double-blind, placebo-controlled trial that included 177 investigative sites in 26 countries and enrolled 10,101 postmenopausal women at increased risk for a major coronary event (primary prevention cohort) or with established CHD (secondary prevention cohort). ${ }^{10}$ Study subjects were followed for a median of 5.6 years. Briefly, eligible women were randomized to receive either raloxifene $60 \mathrm{mg} /$ day $(n=5044)$ or placebo $(n=5057)$. Scheduled follow-up visits occurred every 6 months. The main study findings have been described in detail elsewhere. ${ }^{5}$

VTE were collected as a secondary end point in RUTH. A reported VTE required clinical symptoms supported by relevant diagnostic studies. The occurrence of VTEs was adjudicated by a committee using the following criteria: (1) a report of a Doppler study showing venous obstruction or (2) a report of a venogram showing venous obstruction. For a definitive diagnosis of pulmonary embolism (PE), supporting documents included either (1) a report of a pulmonary angiogram showing PE or (2) a report of a ventilation perfusion $(\mathrm{V} / \mathrm{Q})$ scan showing high probability for (PE). For cases where documentation was unavailable, a likely diagnosis of VTE was based on clinical diagnosis without confirmed procedures. Women diagnosed with a VTE were discontinued from the study drug but encouraged to stay in the trial for continued follow-up off drug.
Patients were allowed to receive standard cardiovascular medications during the study at the discretion of their primary physician. Information on use of other medications was collected from patients at baseline and at each study visit, which occurred every 6 months. Concomitant medications were grouped into categories using the Anatomical Therapeutic Chemical (ATC) codes. Data on the use of antiplatelet medications, including aspirin or nonaspirin antiplatelet agents (clopidogrel, ticlopidine, and dipyridamole), were used for this analysis. Antiplatelet users included women who reported any use of antiplatelet agents after baseline. This encompasses both women who were taking antiplatelet agents at baseline and continued during the study and new users of antiplatelet agents who initiated use during the study.

\section{Statistical analysis}

Baseline characteristics between treatment groups were compared using two-group $t$ test for continuous variables and chi-square test for categorical variables. Antiplatelet agent use in the analyses was restricted to use at baseline, any use during study period, or use before a VTE occurrence. Antiplatelet agents were categorized as aspirin and nonaspirin, which includes clopidogrel, ticlopidine, and dipyridamole.

Main analyses evaluated the effect of any concomitant antiplatelet agent use on VTE risk in women taking raloxifene and placebo. Raloxifene effects on VTE risk were assessed separately for women who used antiplatelet agents and for women who did not use antiplatelet agents during the study period. The effects of concomitant aspirin and nonaspirin use on VTE risk were also assessed. VTE risks were evaluated for those who used antiplatelet agents before study enrollment (at baseline) and at any time during the study period. Analyses were also performed in subgroups of women at risk of CHD (primary prevention cohort) and those with established CHD (secondary prevention cohort) at study entry. Cox proportional hazard models were used, with time from randomization to first VTE as an outcome and use of warfarin, presence of fracture, and hospitalizations, either during the study period or before first VTE occurrence, as covariates. Hazard ratios (HR) and 95\% confidence intervals (CI) were estimated from the models. Unadjusted Cox proportional hazard models are used for baseline-related analyses. All statistical analyses were performed with SAS software version 8.2 (SAS Institute).

\section{Results}

Baseline characteristics of the RUTH study population have been published in detail previously. ${ }^{10}$ Briefly, the mean age

Table 1. Use of Antiplatelet Agents in Raloxifene Use for the Heart Trial

\begin{tabular}{|c|c|c|c|c|}
\hline & \multicolumn{2}{|c|}{ Baseline } & \multicolumn{2}{|c|}{ During study } \\
\hline & Placebo n (\%) & Raloxifene n (\%) & Placebo n (\%) & Raloxifene n (\%) \\
\hline AP use & 2967 (58.7) & $2960(58.7)$ & 3709 (73.3) & 3759 (74.5) \\
\hline Aspirin use & $2865(56.7)$ & $2846(56.4)$ & 3545 (70.1) & 3606 (71.5) \\
\hline Nonaspirin AP use & $142(2.8)$ & $156(3.1)$ & 733 (14.5) & 704 (14.0) \\
\hline
\end{tabular}

$\mathrm{AP}$, antiplatelet agent. 
Table 2. Baseline Characteristics in Women Who Used or Did Not Use Antiplatelet Agents in Raloxifene Use for the Heart Trial

\begin{tabular}{lcr}
\hline Characteristic & AP user $(\mathrm{n}=7468)^{\mathrm{a}}$ & Not \pm AP user $(\mathrm{n}=2625)$ \\
\hline Age (years, mean $\pm \mathrm{SE})$ & $67.7 \pm 6.6$ & $66.9 \pm 6.7$ \\
BMI $\left(\mathrm{kg} / \mathrm{m}^{2}\right.$, mean \pm SE) & $28.6 \pm 5.1$ & $29.2 \pm 5.4$ \\
Diabetes mellitus (\%) & 40.9 & 59.5 \\
Hypertension (\%) & 76.0 & 83.1 \\
Hyperlipidemia (\%) & 75.4 & 67.7 \\
History of CHD (\%) & 60.8 & 18.9 \\
Prior myocardial infarction (\%) & 35.2 & 12.3 \\
Lower extremity arterial disease (\%) & 11.5 & 8.5 \\
\hline
\end{tabular}

${ }^{a}$ All comparisons between AP users and AP nonusers were significant $(p<0.01)$.

BMI, body mass index; CHD, coronary heart disease; SE, standard error.

for the women entering the study was 67.5 years, with $39 \%$ being $\geq 70$ years of age. Approximately $50 \%$ of the women had prior CHD events, $78 \%$ had hypertension, and $46 \%$ had diabetes. ${ }^{10}$ There were no statistically significant differences between the raloxifene and placebo groups with respect to cardiovascular risk factors and VTE risk factors. In 1998 when the trial began, slightly more than half $(59 \%)$ of the study participants were taking antiplatelet agents at baseline, with the vast majority of that antiplatelet agent use occurring in the form of aspirin (Table 1). During the course of the study, more women began using antiplatelet agents, so that by study end, almost $74 \%$ of the women were using an antiplatelet agent (Table 1). Women using antiplatelet agents in the study were significantly older, more likely to have a history of CHD and prior myocardial infarction (MI), less likely to have diabetes or hypertension, and more likely to have hyperlipidemia compared with women not using antiplatelet agents (Table 2). There were no differences between the placebo and raloxifene groups in the proportions of women who used or did not use antiplatelet agents.

For the study population as a whole, there was a significant increase in the risk of VTE in the women randomized to raloxifene (103 VTE events in 5044 patients, 2.0\%) compared with those assigned to placebo (71 VTE events in 5057 patients, $1.4 \%$ ) (HR 1.44, 95\% CI 1.06-1.95), as shown in the RUTH results published earlier. ${ }^{5}$ Table 3 gives the number of VTEs by subject group. Antiplatelet agent nonusers had a lower VTE risk, reflecting their differing risk profiles. The cumulative incidence curves of VTE per 1000 woman-years were similar for antiplatelet agent users and nonusers in both treatment groups (Fig. 1). Raloxifene increased VTE risk compared with placebo, and the increased risks were not different between women who used antiplatelet agents during the study period and those who did not (HR 1.40 vs. 1.54, interaction $p=0.82$ ) (Fig. 2). The vast majority of antiplatelet agent use consisted of aspirin (Table 1). Hence, when antiplatelet agents were separated (Fig. 3) into aspirin and nonaspirin agents (clopidogrel, ticlopidine, and dipyridamole), the use of aspirin (Fig. 3A) was associated with similar results compared with antiplatelet agents as a whole (Fig. 2). There was no significant difference in the increased risk of VTE with raloxifene between aspirin use and no aspirin use (interaction $p=0.85$ ). The raloxifene-associated increase in VTE risk was not changed with the use of nonaspirin antiplatelet agents (Fig. 3B) (interaction $p=0.79$ ). The majority of VTEs were deep venous thromboses (DVT); Table 4 provides a breakdown of VTEs by subtype.

The effect of raloxifene use on VTE risk, compared with placebo, was not different in women who used antiplatelet agents at baseline (HR 1.44, 95\% CI 0.98-2.10) and those who did not use antiplatelet agents at baseline (HR 1.37, 95\% CI 0.83-2.27 (interaction $p=0.88$ ). The increase in VTE risk with raloxifene compared with placebo was also not different between women who used antiplatelet agents after baseline (HR 1.74, 95\% CI 1.10-2.73) and those who did not use antiplatelet agents (HR 1.24, 95\% CI 0.82-1.86) (interaction $p=0.29$ ). In women at risk of CHD (primary prevention cohort), no decrease in raloxifene-associated VTE risk with antiplatelet use was observed (Fig. 4A). However, in women with established CHD (secondary prevention cohort), the increase in VTE risk among antiplatelet agent users (HR 1.11, 95\% CI 0.72-1.70) appeared to be lower when compared with nonusers of antiplatelet agents (HR 2.09, 95\% CI 0.41-10.78), although the difference was not statistically significant (interaction $p=0.48$, (Fig. 4B). The reason for an apparently lower VTE risk among antiplatelet agent users in the secondary prevention cohort was that there was a higher VTE risk among study participants randomized to the placebo arm who used antiplatelet agents.

\section{Discussion}

The purpose of this post hoc analysis of the RUTH study population was to determine if concomitant use of antiplatelet agents by women in the trial could attenuate the heightened risk of VTEs known to exist with the use of the SERM raloxifene. For this analysis, we captured use of antiplatelet agents at baseline and new use at any point during the study. Many

Table 3. Venous Thromboembolic Events by Subject Subgroup

\begin{tabular}{llc}
\hline & $\begin{array}{c}\text { AP user } \\
\mathrm{n}=7468\end{array}$ & $\begin{array}{c}\text { Not AP } \\
\text { user } \mathrm{n}=2625\end{array}$ \\
\hline $\begin{array}{l}\text { Raloxifene group } n=5038 \\
\text { Placebo group } n=5055\end{array}$ & $55(1.5 \%)$ & $25(0.5 \%)$ \\
\hline
\end{tabular}

Percentages are given as percent of treatment group, that is, raloxifene group or placebo group. 

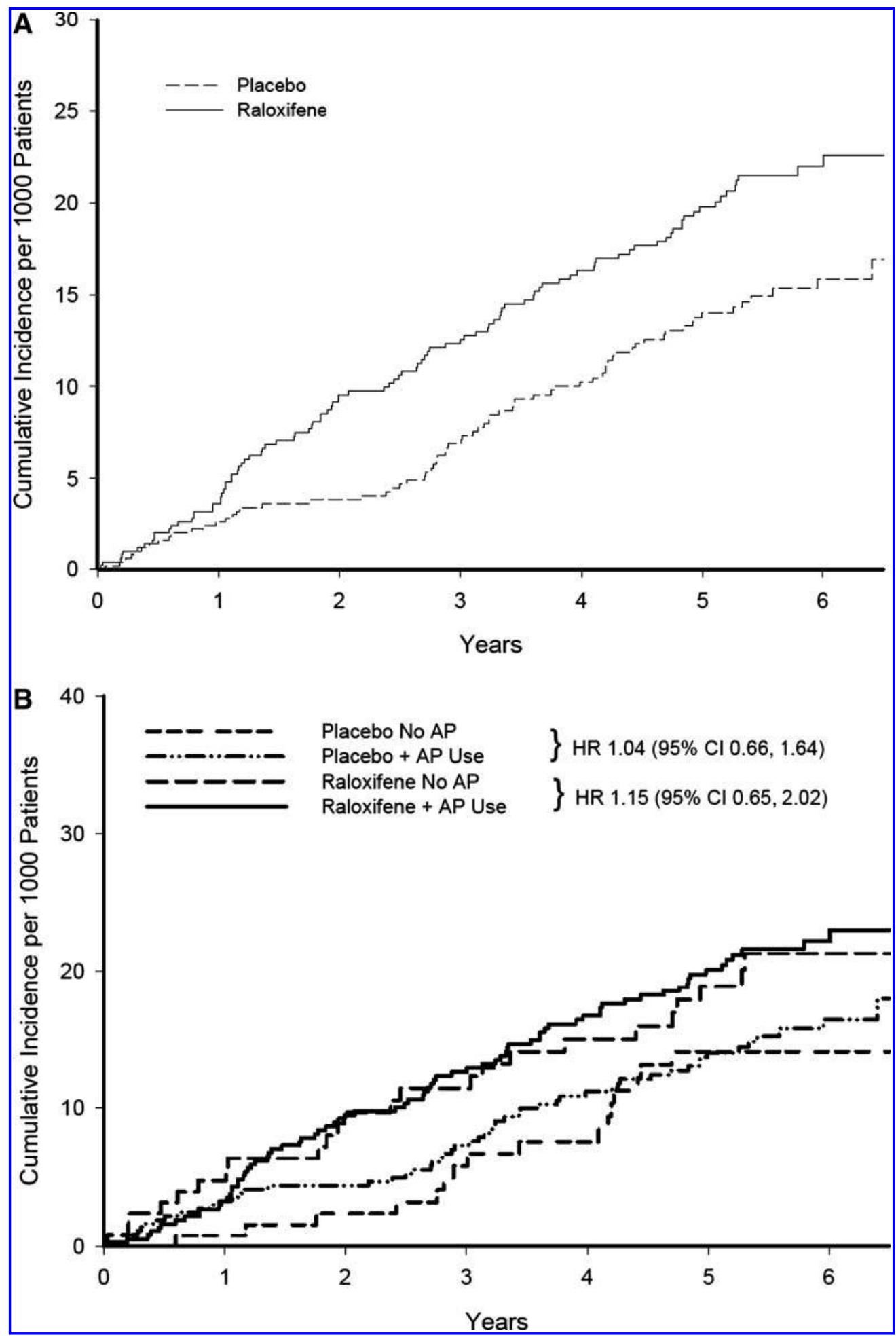

FIG. 1. Kaplan-Meier graphs showing the cumulative incidence of venous thromboembolism (VTE) events in postmenopausal women in the Raloxifene Use for the Heart (RUTH) study. (A) Overall incidence of VTE in the placebo ( $n=5057)$ and raloxifene $(n=5044)$ treatment groups. The risk of VTE was significantly greater in the raloxifene group compared with the placebo group (HR 1.44, 95\% CI 1.06-1.95). (B) The incidence of VTE in women who used antiplatelet agents was similar to that seen in women with no antiplatelet agent use in both the placebo and raloxifene treatment groups. HR, hazard ratio; CI, confidence interval. 


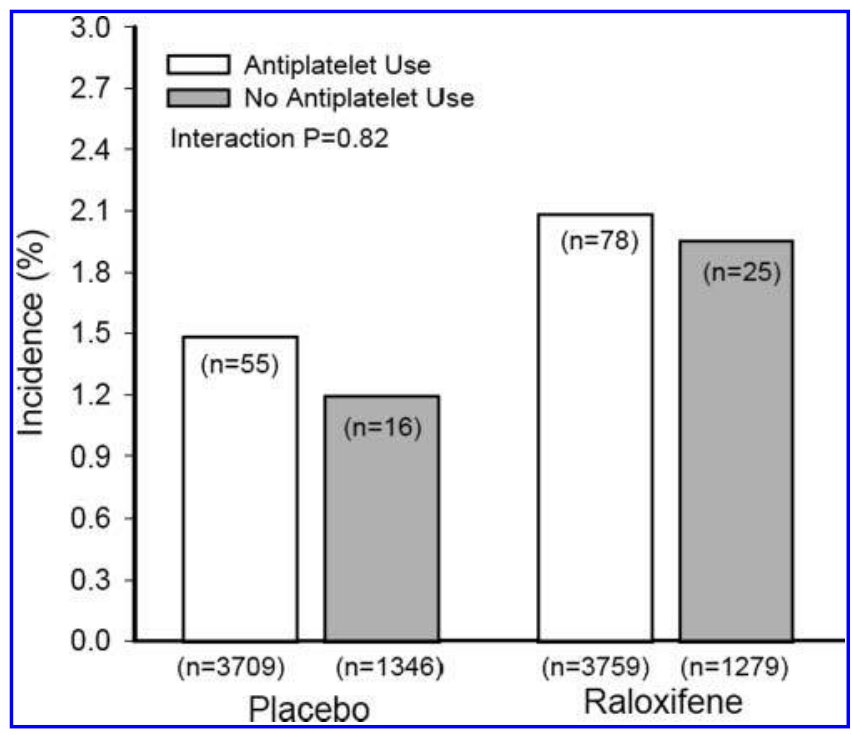

FIG. 2. The risks of VTE were increased with raloxifene compared with placebo in postmenopausal women who used antiplatelet agents during the RUTH study and in those who did not use antiplatelet agents. A total of 174 VTEs were observed in the study, as shown by the numbers within the bars. The numbers below the bars indicate the total numbers of women in each subgroup. The increased risks were similar between the antiplatelet agent use subgroups. $p$ values indicate the treatment by antiplatelet agent use interaction. VTE, venous thromboembolism events.

women initiated antiplatelet agent use during the course of the study, presumably for prophylactic use because of cardiovascular disease risk or occurrence. Although we cannot know for sure, it is reasonable to expect that most women, once antiplatelet agent use was recommended to them and initiated, would continue to take the agents on an ongoing basis. VTEs, including PEs, are uncommon but serious and potentially fatal events in the general population, occurring with an incidence of approximately 1 adult per 1000 personyears. ${ }^{11}$ The use of oral estrogen as well as SERMs has been shown to increase the risk of VTE. ${ }^{3,12,13}$ Oral estrogen is thought to induce first-pass effects, causing alterations in coagulation factor production or metabolism and leading to possible prothrombotic effects. ${ }^{14}$ The SERMs are thought to increase VTE risk by similar mechanisms, ${ }^{6}$ although the precise changes in hemostatic balance responsible for increased VTEs are not fully understood. VTEs are associated with reproducible conditions, such as recent surgery or prolonged immobilization, and with increased blood coagulability resulting from either acquired conditions (e.g., cancer) or inherited genetic variants. ${ }^{15}$ People known to have these risk factors should not receive oral estrogen or SERM therapy because of the heightened risk of subsequent venous events.

Strategies aimed at preventing VTEs include such mechanical interventions as compression stockings and intermittent pneumatic compression, early mobilization after surgery, and prophylactic antithrombotic agents, such as intravenous or subcutaneous unfractionated heparin, subcutaneous low molecular weight heparin, and oral warfarin. Antiplatelet agent therapy with aspirin or an adenosine diphosphate inhibitor has not been a recommended strategy for
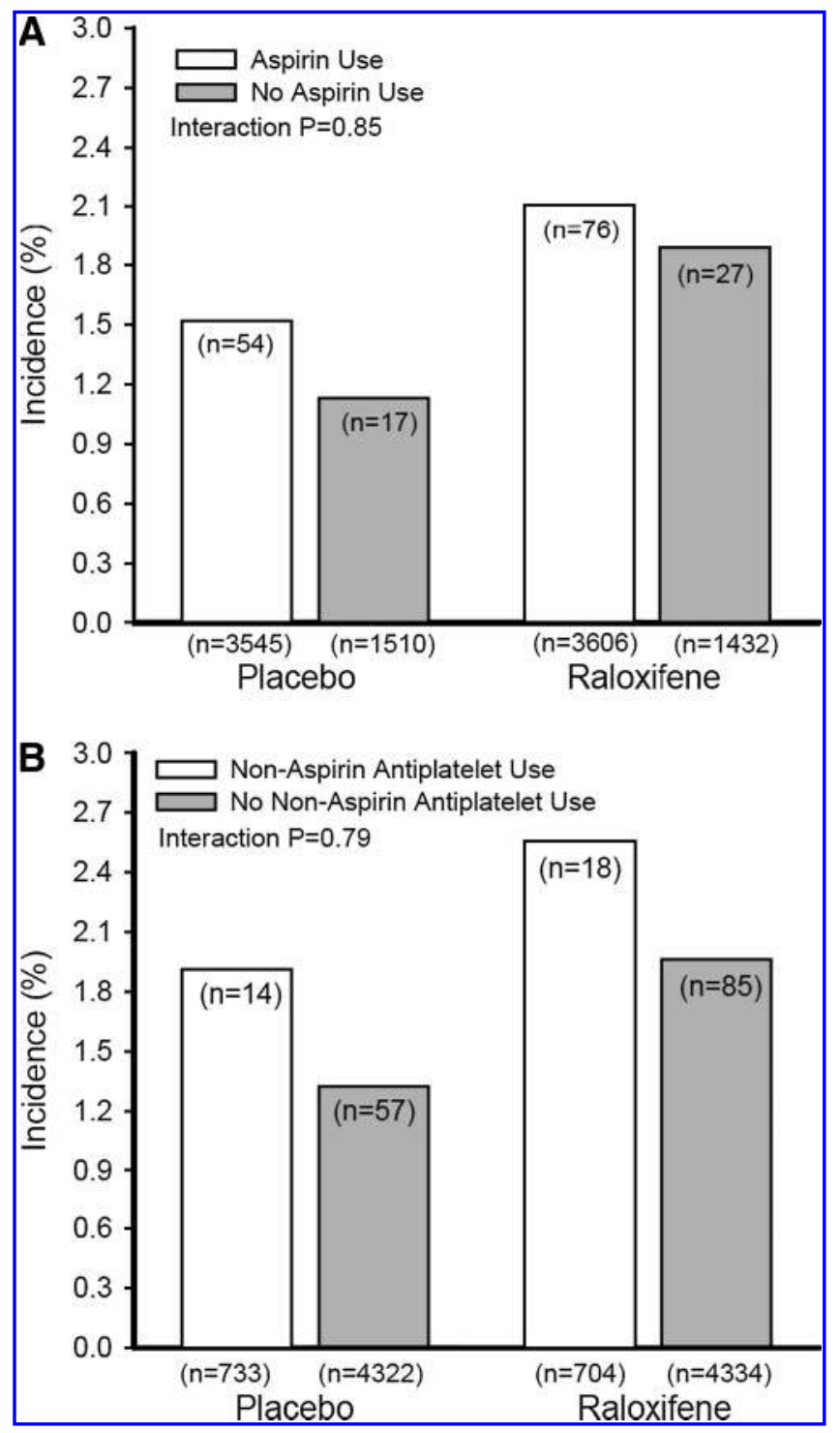

FIG. 3. The risks of VTE were increased with raloxifene compared with placebo in postmenopausal women who did or did not use (A) aspirin or (B) nonaspirin antiplatelet agents during the RUTH study. The increased risks were similar between the subgroups who did or did not use aspirin (A) and between the subgroups who did or did not use nonaspirin antiplatelet agents (B). Numbers within the bars indicate the numbers of VTEs, and numbers below the bars indicate the total numbers of women in each subgroup. The increased risks were not statistically significant. $p$ values indicate the treatment by antiplatelet agent use interaction.

VTE prevention because venous thrombi consist primarily of red cells and fibrin, not platelets. ${ }^{16}$ Pathophysiological relationships exist between arterial and venous thrombi, however, and platelet activation does play a role in the generation and propagation of thrombin even in venous thromboses. ${ }^{16}$ Recent large studies have shown an interrelationship between atherosclerotic heart disease, MI, and VTE risk. ${ }^{17,18}$ Several antiplatelet agent trials have shown a modest decrease in VTE risk associated with aspirin therapy, on the order of $30 \%$, although the risk reduction was substantially less than that associated with antithrombotic therapy. ${ }^{19,20}$ Furthermore, 
Table 4. Venous Thromboembolic Events by Type (Individuals May Have Deep Venous Thrombosis and Pulmonary Embolism)

\begin{tabular}{lcccc}
\hline & $\begin{array}{c}\text { Raloxifene }+ \text { AP } \\
\mathrm{n}=3759\end{array}$ & $\begin{array}{c}\text { Raloxifene, no } \\
\text { AP n=1279 }\end{array}$ & $\begin{array}{c}\text { Placebo }+ \text { AP } \\
\mathrm{n}=3709\end{array}$ & $\begin{array}{c}\text { Placebo, no } \\
\text { AP } \mathrm{n}=1346\end{array}$ \\
\hline DVT & 47 & 18 & 39 & 8 \\
PE & 28 & 8 & 16 & 8 \\
Intracranial thrombosis & 6 & 2 & 3 & 3 \\
Other & 2 & 0 & 0 & 1 \\
\hline
\end{tabular}

DVT, deep venous thrombosis; PE, pulmonary embolism.
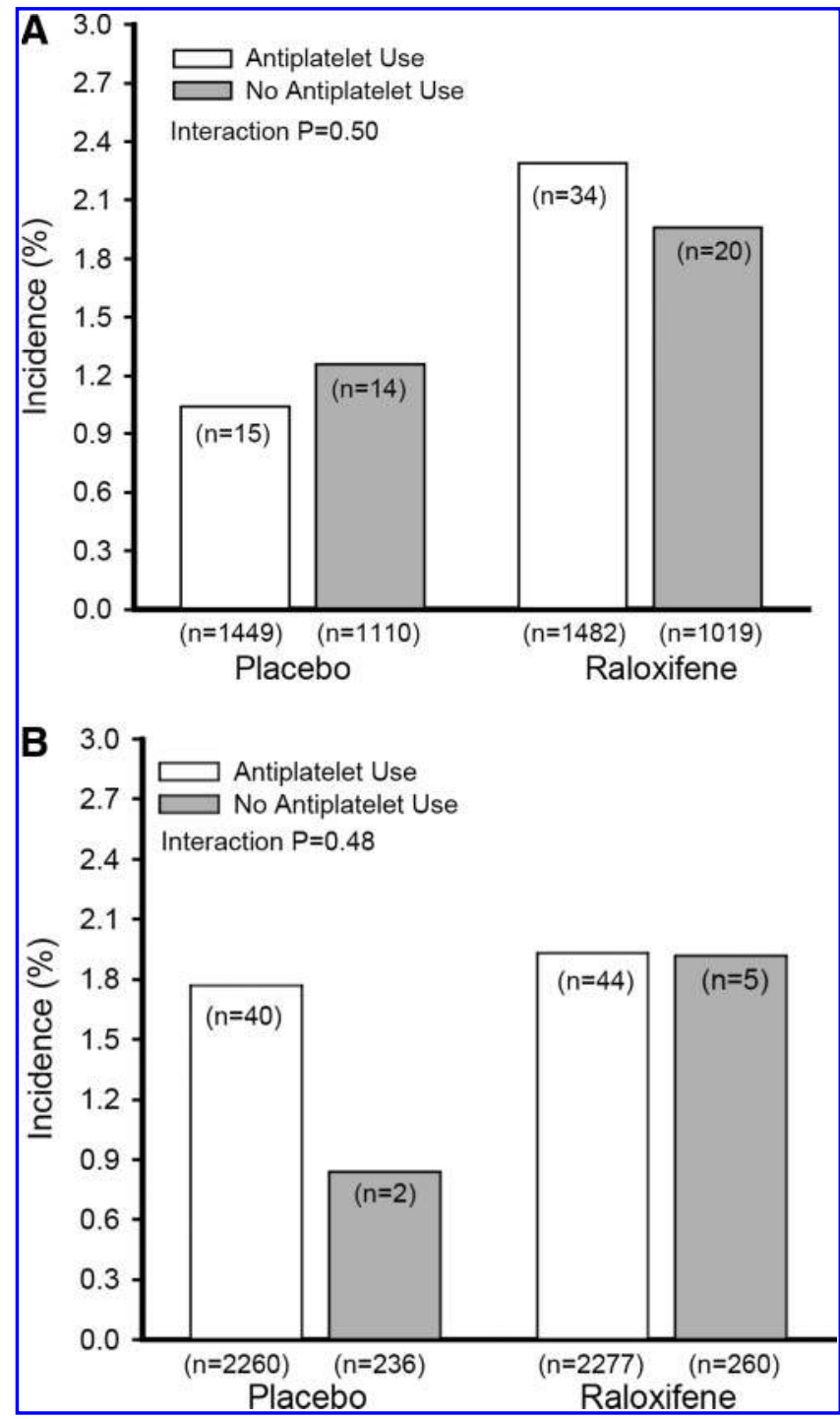

FIG. 4. The risks of VTE were increased with raloxifene compared with placebo in subgroups of women who used and did not use antiplatelet agents during the RUTH study for cohorts of postmenopausal women (A) at increased risk of coronary heart disease (primary prevention) and (B) with established CHD (secondary prevention). The increased risks with raloxifene treatment were similar in the primary $(\mathbf{A})$ and secondary (B) prevention cohorts between the subgroups who did or did not use antiplatelet agents. Numbers within the bars indicate the numbers of VTEs, and numbers below the bars indicate the total numbers of women in each subgroup. The increased risks were not statistically significant. $p$ values indicate the treatment by antiplatelet agent use interaction. analysis of the Heart and Estrogen/progestin Replacement Study Follow-up (HERS II) adverse events showed that baseline aspirin use in that study was (weakly) associated with an attenuated risk of VTE. ${ }^{21}$ In the WHI, however, a large, primary prevention trial of oral estrogen and MPA in healthy postmenopausal women, no VTE risk reduction was found for women in the study using antiplatelet agents. ${ }^{22}$

The results from these current analyses failed to show any diminution of raloxifene-related VTE risk with concomitant use of antiplatelet agents for the women enrolled in the RUTH trial. Neither aspirin nor nonaspirin antiplatelet agents reduced VTE risk. The women studied in RUTH were, on average, 10 years postmenopause and were either at high risk for developing CHD or already had established CHD. Users of antiplatelet agents differed from nonusers; they were far more likely to have prior CHD and MIs, had a higher VTE risk, and were less likely to have diabetes and hypertension. As the study did not control for use of other medications, such as statins, which have been shown recently to be associated with decreased VTE risk, it is impossible to ascertain whether antiplatelet use per se had any effect on VTE risk. ${ }^{23}$ This should be analyzed in future studies.

\section{Study limitations}

This was a post hoc exploratory analysis of a large, randomized trial conducted to examine an auxiliary end point. The primary end point, VTE risk, could have been overestimated, as VTEs could be self-reported in the trial without medical documentation. The majority of subjects $(\sim 74 \%)$ took an antiplatelet agent during the study, but the duration of use was not assessed. Antiplatelet agent use was not randomized in the trial; however, use did not differ between the treatment groups, and potential confounders were balanced between treatment groups within subgroups of women who used or did not use antiplatelet agents. It must be noted that information on concomitant medication use collected as typically done in a clinical trial should be considered to be potentially unreliable, especially regarding start and stop dates.

\section{Conclusions}

In the RUTH study overall, the use of antiplatelet agents was not associated with a reduced incidence of VTE in either the placebo or raloxifene group. The increased risk of VTE with raloxifene compared with placebo was not influenced by the use of antiplatelet agents (aspirin or nonaspirin). Further studies of potential protective effects of antiplatelet agents against VTE are warranted in the secondary prevention subgroup of women. 


\section{Acknowledgments}

This work was supported by grants from Eli Lilly \& Company.

\section{Disclosure Statement}

C.S.D. is on the Speakers Bureau of Pfizer, Inc., and has received study funding from Pfizer Inc. and the Veteran's Administration. The other authors are employees of Eli Lilly \& Company.

\section{References}

1. Eli Lilly and Company: Evista prescribing information, 1997.

2. Canonico M, Plu-Bureau G, Lowe GD, Scarabin PY. Hormone replacement therapy and risk of venous thromboembolism in postmenopausal women: Systematic review and meta-analysis. BMJ 2008;336:1227-1231.

3. Grady D, Wenger NK, Herrington D, et al. Postmenopausal hormone therapy increases risk for venous thromboembolic disease: The Heart and Estrogen/progestin Replacement Study. Ann Intern Med 2000;132:689-696.

4. Grady D, Ettinger B, Moscarelli E, et al. Safety and adverse effects associated with raloxifene: Multiple outcomes of raloxifene evaluation. Obstet Gynecol 2004;104:837-844.

5. Barrett-Connor E, Mosca L, Collins P, et al. Effects of raloxifene on cardiovascular events and breast cancer in postmenopausal women. N Engl J Med 2006;355:125-137.

6. Blumenthal RS, Baranowski B, Dowsett SA. Cardiovascular effects of raloxifene: The arterial and venous systems. Am Heart J 2004;147:783-789.

7. Sumino H, Ichikawa S, Kasama S, et al. Different effects of oral conjugated estrogen and transdermal estradiol on arterial stiffness and vascular inflammatory markers in postmenopausal women. Atherosclerosis 2006;189:436-442.

8. Hulley S, Grady D, Bush T, et al. Randomized trial of estrogen plus progestin for secondary prevention of coronary heart disease in postmenopausal women. Heart and Estrogen/progestin Replacement Study (HERS) Research Group. JAMA 1998;280:605-613.

9. Glynn RJ, Ridker PM, Goldhaber SZ, Buring JE. Effect of lowdose aspirin on the occurrence of venous thromboembolism: A randomized trial. Ann Intern Med 2007;147:525-533.

10. Mosca L, Barrett-Connor E, Wenger NK, et al. Design and methods of the Raloxifene Use for The Heart (RUTH) study. Am J Cardiol 2001;88:392-395.

11. World Health Organization Collaborative Study of Cardiovascular Disease and Steroid Hormone Contraception. Venous thromboembolic disease and combined oral contraceptives: Results of international multicentre case-control study. Lancet 1995;346:1575-1582.

12. Cummings SR, Eckert S, Krueger KA, et al. The effect of raloxifene on risk of breast cancer in postmenopausal women:
Results from the MORE randomized trial. Multiple Outcomes of Raloxifene Evaluation. JAMA 1999;281:2189-2197.

13. Fisher B, Costantino JP, Wickerham DL, et al. Tamoxifen for prevention of breast cancer: Report of the National Surgical Adjuvant Breast and Bowel Project P-1 Study. I Natl Cancer Inst 1998;90:1371-1388.

14. Meilahn EN. Hemostatic factors and risk of cardiovascular disease in women. An overview. Arch Pathol Lab Med 1992; 116:1313-1317.

15. Turpie AG, Chin BS, Lip GY. Venous thromboembolism: Pathophysiology, clinical features, and prevention. BMJ 2002;325:887-890.

16. Schafer AI. Antiplatelet therapy. Am J Med 1996;101:199_ 209.

17. Sørensen HT, Horvath-Puho E, Pedersen L, Baron JA, Prandoni P. Venous thromboembolism and subsequent hospitalisation due to acute arterial cardiovascular events: A 20-year cohort study. Lancet 2007;370:1773-1779.

18. Prandoni P, Bilora F, Marchiori A, et al. An association between atherosclerosis and venous thrombosis. N Engl J Med 2003;348:1435-1441.

19. Antiplatelet Trialists' Collaboration. Collaborative overview of randomised trials of antiplatelet therapy. I: Prevention of death, myocardial infarction, and stroke by prolonged antiplatelet therapy in various categories of patients. BMJ 1994;308:81-106.

20. Prevention of pulmonary embolism and deep vein thrombosis with low dose aspirin: Pulmonary Embolism Prevention (PEP) Trial Collaborative Group. Lancet 2000; 355:1295-1302.

21. Hulley S, Furberg C, Barrett-Connor E, et al. Noncardiovascular disease outcomes during 6.8 years of hormone therapy: Heart and Estrogen/progestin Replacement Study follow-up (HERS II). JAMA 2002;288:58-66.

22. Cushman M, Kuller LH, Prentice R, et al. Estrogen plus progestin and risk of venous thrombosis. JAMA 2004;292: 1573-1580.

23. Glynn RJ, Danielson E, Fonseca FA, et al. A randomized trial of rosuvastatin in the prevention of venous thromboembolism. N Engl J Med 2009;360:1851-1861.

Address correspondence to: Claire S. Duvernoy, M.D. Associate Professor of Medicine University of Michigan Health System Chief, Cardiology Section Veterans Affairs Ann Arbor Healthcare System 2215 Fuller Road, Box 111a Ann Arbor, MI 48105-2399

E-mail: duvernoy@umich.edu 
